\title{
CÓDIGOS PARA O CANAL ADITIVO COM DOIS USUÁRIOS BINÁRIOS
}

\author{
Maria de Lourdes M.G. Alcoforado e Valdemar Cardoso da Rocha Junior
}

\begin{abstract}
Resumo - Códigos para o canal aditivo com dois usuários binários (2-BAC) são investigados neste trabalho, na situação em que o código empregado por um dos usuários é linear e não há ruído no sistema. Especificamente é apresentada uma dedução mais simples para a taxa de transmissão de códigos lineares fortemente ortogonais, são introduzidos os códigos lineares fortemente ortogonais balanceados e os códigos de paridade repetida, com as respectivas taxas de transmissão. $\mathrm{O}$ algoritmo de Cabral, para a construção linear de códigos para o 2-BAC. foi implementado pela primeira vez e alguns dos códigos construídos são aqui apresentados.
\end{abstract}

Abstract - Coding for the two-user binary adder channel (2-BAC) is investigated for the situation where one of the two codes is linear and the channel is noiseless. A simpler derivation of the transmission rate of linear strongly orthogonal codes is presented. Linear balanced strongly orthogonal codes are introduced, as well as repeated parity codes, together with their respective transmission rates. The Cabral algorithm for the linear construction of codes for the 2-BAC was implemented for the first time and a few of the codes obtained are presented in this paper.

Palavras-chave: Canais aditivos, codificação, códigos para o 2-BAC

\section{INTRODUÇÃO}

O mais simples modelo de canal de acesso múltiplo é o representado pelo canal aditivo com dois usuários binários (2-BAC), na ausência de ruído [1]. Neste sistema dois usuários geograficamente separados tentam enviar dados binários através de um mesmo canal de comunicações. A função utilizada neste canal para combinar os dois usuários é a adição sobre os reais, onde cada usuário tem como alfabeto o conjunto $F_{2}=(0,1)$. A entrada do canal consiste. portanto, de duplas binárias $x_{i}, w_{i}$ e a saída $y_{i}=x_{i}+w_{i}$. consiste de símbolos do alfabeto $\{0,1,2\}$.

Um dos principais objetivos dos pesquisadores que investigam o 2-BAC é obter códigos unicamente decodificáveis com taxas de transmissão tão próximas à fronteira da região de capacidade [1] quanto possível. Cabral [2] determinou um algoritmo para, a partir de um código $C_{1}$ linear específico, produzir um código $C_{2}$ com o maior número possível de palavrascódigo. Mais especificamente, Cabral determinou condições

O trabalho de Valdemar Cardoso da Rocha Junior foi parcialmente financiado pelo Conselho Nacional de Desenvolvimento Científico e Tecnológico (CNPq). Projeto No. 304214/77-9.

Os autores são do Grupo de Pesquisa em Comunicações - CODEC. Departamento de Eletrônica e Sistemas, Universidade Federal de Pernambuco, CP 7800. CEP 50.711-970 Recife PE BRASIL. Tel.: (81) 3271-8210, Fax: (81) 3271-8215 que dois conjuntos de vetores binários devem satisfazer para garantir sua decodibilidade única sobre o 2-BAC, dando ênfase ao caso em que um dos conjuntos de vetores é um código de bloco linear. Os resultados obtidos por Cabral permitem dividir a busca de vetores para $C_{2}$ no espaço das $n$ uplas binárias, i.e., $C_{2} \in F_{2}^{n}$, em buscas em subconjuntos de $F_{2}^{n}$, de menor cardinalidade e independentes entre si.

Na Seção 2 apresentamos a caracterização do 2-BAC, tratando da decodibilidade única e dos códigos lineares para o 2-BAC. Na Seção 5 apresentamos uma revisão sucinta de códigos fortemente ortogonais [2] e uma dedução mais simples para a taxa de transmissão dos mesmos. Nas Seçôes 7 e 8 introduzimos os códigos lineares fortemente ortogonais balanceados e os códigos de paridade repetida [3], respectivamente, acompanhados de expressões analíticas para as correspondentes taxas de transmissão. Na Seção 9 apresentamos alguns dos códigos obtidos da construção linear de Cabral, implementada pela primeira vez. Na Seção 10 é mostrado como particionar em dois subconjuntos o dicionário das palavras de um código, de modo a formar um par unicamente decodificável para o 2-BAC. Finalmente na Seção 11 apresentamos as conclusões finais e alguns comentários.

\section{CANAL 2-BAC}

Consideramos no que segue que os dois usuários utilizam códigos binários de mesmo comprimento $n$, operam na mesma freqüencia. transmitem ao mesmo tempo, operam com sincronismo de palavra e existe um único decodificador. Supomos que os dois usuários escolhem independentemente as respectivas palavras-código a serem transmitidas. O usuário 1 envia palavras-código de um código de bloco $C_{1}$; o usuário 2 envia palavras-código de um código de bloco $C_{2}$. Representamos por $\left(C_{1}, C_{2}\right)$ um código de comprimento $n$ para dois usuários, onde o código constituinte $C_{1}$ tem $L$ palavras $\left\{x_{1}, x_{2} \ldots \ldots, x_{L}\right\}$ e o código constituinte $C_{2}$ tem $M$ palavras $\left\{w_{1}, w_{2}, \ldots, w_{M}\right\}$. A taxa de transmissão conjunta $R$ do par $\left(C_{1}, C_{2}\right)$ é dada por:

$$
R=R_{1}+R_{2}=\frac{\log _{2} L}{n}+\frac{\log _{2} M}{n}
$$

Para pontos $\left(R_{1}, R_{2}\right)$ dentro da região de capacidade do canal, existem os codificadores e o decodificador tais que cada remetente pode se comunicar com o receptor com probabilidade de erro zero ou muito pequena.

\section{DECODIBILIDADE ÚNICA}

Consideremos uma extensão para o 2-BAC onde cada entrada é uma $n$-upla binária, denotadas respectivamente por $\mathbf{x}$ 
e w sendo

$$
\begin{aligned}
\mathbf{x} & =\left(x_{1}, x_{2}, \ldots, x_{n}\right) \\
\mathbf{w} & =\left(w_{1}, w_{2}, \ldots, w_{n}\right)
\end{aligned}
$$

a saída para o 2-BAC será então,

$$
\mathbf{y}=\left(x_{1}+w_{1}, x_{2}+w_{2}, \ldots x_{n}+w_{n}\right)
$$

Definindo as operações de adição e multiplicação binárias sobre $n$-uplas binárias como as respectivas operações sobre $F_{2}$, aplicadas componente a componente temos que.

$$
\begin{aligned}
\mathbf{x} \oplus \mathbf{w} & =\left(x_{1} \ominus w_{1}, x_{2} \oplus w_{2}, \ldots, x_{n} \sqcup w_{n}\right) \\
\mathbf{x} . \mathbf{w} & =\left(x_{1} . w_{1}, x_{2} . w_{2}, \ldots, x_{n} . w_{n}\right)
\end{aligned}
$$

Nas referências [2] e [4] foi mostrado que

$$
\mathrm{x}+\mathrm{w}=\mathrm{x} \cdot \mathrm{w}+2 \mathrm{x} \cdot \mathrm{w},
$$

resultado este que permitiu tratar a síntese de códigos binários para o 2-BAC considerando apenas as operações lógicas $€$ (ou-exclusivo) e . (e-lógico). Nosso interesse no canal 2-BAC é construir um par de códigos $C_{1}$ e $C_{2}$ de modo que:

Condição 1 O decodificador deve ser capa: de decodificar o vetor $\mathrm{y}$ recebido, sem ambigüidade, nas duas palavrascódigo que foram transmitidas pelos usuários l e 2. Isto é, se para quaisquer $\mathbf{x}_{1} \in C_{1}, \mathbf{x}_{2} \in C_{1}$ e $\mathbf{w}_{1} \in C_{2}, \mathbf{w}_{2} \in C_{2}$ tais que $\mathbf{x}_{1} \neq \mathbf{x}_{2}$, e $\mathbf{w}_{\mathbf{1}} \neq \mathbf{w}_{\mathbf{2}}$, então $\mathbf{x}_{1}+\mathbf{w}_{\mathbf{1}} \neq \mathbf{x}_{\mathbf{2}}+\mathbf{w}_{\mathbf{2}}$. Condição 2 As taxas $\left(R_{1}, R_{2}\right)$, respectivamente de $C_{1}$ e $C_{2}$, devem situar-se dentro da região de capacidade e tão próxinuas à fronteira quanto possível.

Um par de códigos $\left(C_{1}, C_{2}\right)$ que satisfaz a Condição $l$ acima é dito ser unicamente decodificável. Eventualmente faremos referência ao par $\left(C_{1}, C_{2}\right)$ como "o código" $\left(C_{1} . C_{2}\right)$.

\section{CÓDIGOS LINEARES}

Um caso de interesse no estudo de códigos para o 2-BAC é aquele em que apenas um dos códigos constituintes é linear.

Definição 1 Um código $\left(C_{1}, C_{2}\right)$ de comprimento $n$ para o 2-BAC é dito ser um código linear se um dos códigos constituintes for um código linear de parâmetros ( $n . k$ ).

$O$ teorema a seguir. provado por Weldon [5], estabelece limitantes para as taxas alcançáveis por códigos lineares.

Teorema 1 Seja $C_{1}$ um código constituinte linear com parâmetros $\left(n, k_{1}\right)$, então a região de capacidade para $u m$ par $\left(C_{1}, C_{2}\right)$ unicamente decodificável é limitada superiormente por $\left(R_{1}, R_{2}\right) \leq\left(k_{1} / n,\left(1-k_{1} / n\right) \log 3\right)$.

Decorre do Teorema $I$ que códigos lineares para o 2 BAC possuem a desvantagem de não atingirem a capacidade quando o código constituinte linear tiver taxa maior que $\left(\log _{2} 3-1\right) / \log _{2} 3$. Rocha [6] observou que na demonstração do referido teorema não é feito uso da linearidade de $C_{1}$ e, portanto, o resultado do Teorema I é válido num contexto mais geral, quando $C_{1}$ é apenas um código sistemático, podendo ser também não linear.

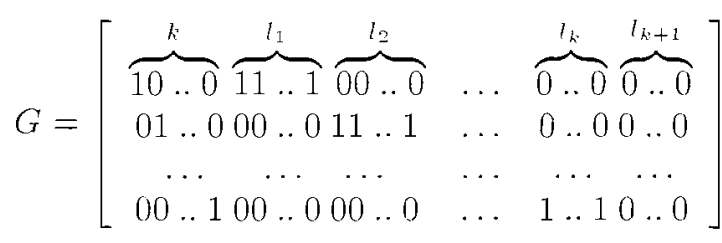

Figura 1. Matriz geradora

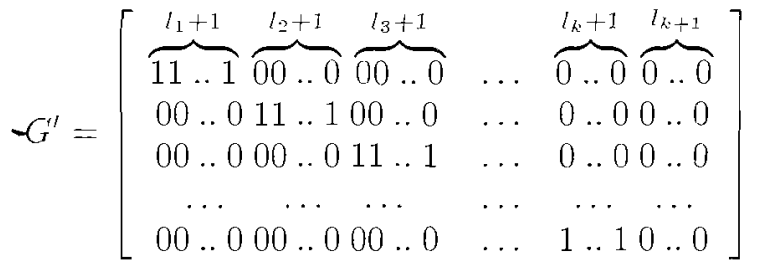

Figura 2. Matriz geradora equivalente

\section{CÓDIGOS FORTEMENTE ORTOGO- NAIS}

Cabral [2] introduziu códigos lineares fortemente ortogonais e determinou analiticamente a taxa de transmissão (vide Teorema 2) destes códigos.

Definição 2 Um código de bloco linear binário $C_{1}$, tendo sua matriz geradora $G$ na forma sistemática, é definido como fortemente ortogonal se para a i-ésima linha de $G$. denotada por $\mathrm{c}_{i}$ e considerada conto $\mathrm{tm}$ vetor, tivernos: $\mathrm{c}_{i} . \mathrm{c}_{j}=0 . i \neq j, i, j \in\{1.2 \ldots k\}$.

Teorema 2 A taxa $R_{2}$ do código $C_{2}$, obtido a partir do código fortemente ortogonal $C_{1}\left(n . h^{2}\right)$ é dada por

$$
R_{2}=\frac{\log _{2}\left(\sum_{i=0}^{k}\left(2^{i} N_{i}\right)+l_{k+1}\right)}{k+\sum_{i=1}^{k} l_{i}+l_{k+1}}
$$

onde os $l_{i}$ 's estão ilustrados na Figura $l$ e $N_{i}$ é dado por

$$
\begin{aligned}
N_{i}= & \sum_{s_{1}=1}^{k} \sum_{s_{2}=s_{1}+1}^{k} \cdots \sum_{s_{i}=s_{i-1}+1}^{k}\left(2^{l_{s_{1}}}-1\right) \times \\
& \left(2^{l_{s_{2}}}-1\right) \times \ldots \times\left(2^{l_{s_{i}}}-1\right) .
\end{aligned}
$$

Apresentamos a seguir uma dedução alternativa e mais simples do Teorema 2

\section{ANÁLISE DE CÓDIGOS FORTEMEN- TE ORTOGONAIS}

É conveniente representarmos a matriz geradora do código linear fortemente ortogonal $C_{1}$ na forma sistemática mostrada na Figura 1. Outra forma de representação da matriz geradora é obtida quando a retiramos da forma sistemática, agrupando todas as $l_{j}, 1 \leq j \leq k+1$, colunas idênticas, inclusive as $k$ colunas mais à esquerda. Podemos visualizar esta representação através da Figura 2.

Neste momento faz-se necessário utilizarmos a construção de Weldon [5] para códigos unicamente decodificáveis, onde 
$0^{n}$ e $1^{n}$ denotam respectivamente as $n$-uplas toda zero e toda 1 .

Construção 1 Seja $C_{1}=\left(0^{n}, 1^{n}\right)$ e seja $C_{2}$ constituído por todas as $n$-uplas exceto $1^{n}$.

Este código é unicamente decodificável pois todas as palavras-código de $C_{2}$ são distintas e todas as $n$-uplas para o 2-BAC serão da forma $\left(0^{n}+c_{2 j}\right)$ ou $\left(1^{n}+c_{2 j}\right)$, onde $c_{2 j}$ denota as palavras-código de $C_{2}$, donde verificamos que $\left(0^{n}+c_{2 j}\right) \cap\left(1^{n}+c_{2 j}\right)=\phi$. O número total de palavrascódigo obtidas para $C_{2}$ é $\left|C_{2}\right|=2^{n}-1$ e o número de palavras-código de $C_{1}$ é $\left|C_{1}\right|=2$. Analisando a matriz da Figura 2 vemos que podemos representá-la como sendo formada por $k+1$ submatrizes geradoras, da seguinte forma

$$
G^{\prime}=\left[G_{1} G_{2} G_{3} \ldots G_{k+1}\right]
$$

Vemos que cada uma das submatrizes geradoras $G_{i} .1 \leq$ $i \leq k$, gera um código de bloco linear com parâmetros $\left(l_{i}, \overline{1}\right)$ e cada uma delas gera duas palavras-código $\left(0^{l_{i}}, 1^{l_{i}}\right)$. Vamos denotar por $C_{1 i}$, o código gerado pela submatriz geradora $G_{i}$ e por $C_{2 i}$, o código encontrado com o máximo número de palavras-código possível, de modo a obtermos a decodibilidade única de $\left(C_{1 i}, C_{2 i}\right)$ no 2-BAC. Observamos que o código $C_{2 i}$ encontrado através do algoritmo implementado é o mesmo obtido pela Construção 1 .

Vamos a partir de agora analisar a submatriz geradora $G_{k+1}$. Vemos que ela gera um código cujo vetor nulo é sua única palavra-código. O código $C_{2, k+1}$ portanto pode ter qualquer uma das $l_{k+1}$-uplas como palavras-código, incluindo o vetor nulo. Portanto o máximo $\left|C_{2 k+1}\right|$ é $2^{l_{k+1}}$. Como temos as $k+1$ submatrizes lado a lado, formando a matriz geradora $G$, as palavras-código de $C_{2}$ resultam da concatenação das palavras-código dos $C_{2 i}$ e portanto o máximo número de palavras-código pertencentes ao código $C_{2}$ é

$$
\left|C_{2}\right|=\prod_{l_{i}=1}^{k}\left(2^{l_{i}}-1\right) 2^{l_{k+1}}
$$

Podemos sintetizar o resultado obtido acima no seguinte teorema.

Teorema 3 A partir do código binário linear fortentente ortogonal $C_{1}$ encontramos um código $C_{2}$, de máximu cardinalidade, contendo $\left|C_{2}\right|=\prod_{l_{i}=1}^{k}\left(2^{l_{i}}-1\right) 2^{l_{k+1}}$ palavrascódigo, de tal modo que o par $\left(C_{1}, C_{2}\right)$ é unicamente decodificável no 2-BAC.

Observamos que os resultados dos Teoremas 2 e 3 e são idênticos. Para taxas $R_{1}$ maiores que $1 / 2$, necessariamente alguns dos $l_{i}$ 's na Figura $l$ serão iguais a zero. Considerando o caso particular em que $l_{k+1}=0$ e cada um dos demais $l_{i}$ "s, $1 \leq i \leq k$, é igual a 1 ou a 0 , resulta que $\sum_{i=1}^{k} l_{i}=n-k \mathrm{e}$ portanto

$$
\left|C_{2}\right|=\left(2^{2}-1\right)^{n-k} .
$$

A taxa $R$ neste caso resulta igual a

$$
R=\frac{k}{n}+\frac{(n-k)}{n} \log _{2} 3
$$

que coincide com o limitante superior do Teorema 1. Introduzimos a seguir os códigos lineares fortemente ortogonais balanceados.

\section{CÓDIGOS FORTEMENTE ORTOGO- NAIS BALANCEADOS}

Definição 3 Seja $C_{1}$ um código de bloco linear sobre $F_{2}$ de parâmetros $(n, k)$, com matriz geradora $G$ na forma sistemática. Denoninando a i-ésima linha da matriz $G$ por $\mathbf{c}_{i}$ e considerando-a cono um vetor em $F_{2}^{n}$, dizemos que $C_{1}$ é un código linear fortemente ortogonal balanceado se $\mathbf{c}_{i} \cdot \mathbf{c}_{j}=0, i, j \in(1,2, \ldots, k)$, com $i \neq j$; e cuja matri- geradora tem a mesma quantidade de 1 's em cada uma de suas linhas.

Uma propriedade interessante de códigos fortemente ortogonais balanceados pode ser observada com o auxilio da Figura 1 , considerando $l_{i}+1=l, 1 \leq i \leq k$ e $l_{k+1}=s \mathrm{e}$ grupando as $k l+s$ colunas em $k$ blocos de $l$ colunas idênticas não-nulas e um bloco com $s$ colunas toda zero. A matriz resultante $G^{\prime}$ gera um código $C_{1}$, equivalente a $C_{1}$ no sentido de que um é obtido do outro através de uma permutação de coordenadas. Aplicando o Teorema 3 para matrizes fortemente ortogonais balanceadas, encontramos que,

$$
\left|C_{2}\right|=\left|C_{2 i}\right|^{k} 2^{s}=\left(2^{l}-1\right)^{k} 2^{s}
$$

pois cada palavra-código de $C_{2}$ será formada pela combinação das $\left(2^{\prime}-1\right)$ palavras-código de cada um dos $C_{2 i}, 1 \leq i \leq k$, e das palavras-código de $C_{2, s}$. Neste caso temos que,

$$
\begin{aligned}
& R_{2}=\frac{\log _{2}\left(2^{l}-1\right)^{k} 2^{s}}{k l+s}=\frac{k}{k l+s} \log _{2}\left(2^{l}-1\right)+\frac{s}{k l+s} \\
& R=R_{1}+R_{2}=\frac{k}{k l+s}+\frac{k}{k l+s} \log _{2}\left(2^{l}-1\right)+\frac{s}{k l+s},
\end{aligned}
$$

que para $s=0$ e $l=2$ reduz-se a

$$
R=\frac{1}{2}+\frac{1}{2} \log _{2} 3
$$

ou seja. para $s=0$ e $l=2$ estes códigos atingem o limitante de Weldon [5] para códigos lineares (Teorenta l).

\section{CÓDIGOS DE PARIDADE REPETIDA}

Um outro caso de interesse é aquele em que a matriz geradora do código $C_{1}$ possui uma propriedade que denominamos de paridade repetida, a qual nos permite calcular o número de palavras-código pertencentes a $C_{2}$.

Definição 4 Códigos de paridade repetida $(n, k)$ são aqueles que possuen metade das palavras-código com os $n-k$ digitos de paridade todos iguais a 0 e a outra metade das palavras-código com os $n-k$ dígitos de paridade todos iguais a 1 .

Exemplo $1 O$ código $C=(10111,01111,11000,00000)$, no qual $n=5$ e $k=2$, é um código de paridade repetida pois, além de linear, tem metade das palavras-código com digitos de paridade 000 e metade com 111, respectivamente.

Nosso objetivo é, a partir da matriz geradora do código de paridade repetida $C_{1}$, determinar o máximo número de 
palavras-código de $C_{2}$, de modo a termos o par $\left(C_{1}, C_{2}\right)$ unicamente decodificável no 2-BAC. O próximo teorema [3] fornece esta resposta.

Teorema 4 Seja $C_{1}$ um código de paridade repetida. Quando a matriz geradora de $C_{1}$ possui

1. um numero par de linhas en que os $n-k$ dígitos de paridade são 1 's, o código $C_{2}$ construído a partir de $C_{1}$ tem um número máximo de $2^{n-k+1}-2$ palarras-código.

2. um número impar de linhas em que os $n-k$ dígitos de paridade são 1 's, o código $C_{2}$ construído a partir de $C_{1}$ ten um número máximo de $2^{n-k+1}-1$ palarras-código.

\section{RESULTADOS}

O algoritmo de Cabral [2] permite encontrar o conjunto de maior cardinalidade de palavras-código para um código $C_{2}$ a partir de um código linear $C_{1}$ dado, de modo a garantir a decodibilidade única no 2-BAC. A nossa implementação do algoritmo de Cabral consiste dos seguintes passos.

1. É solicitada a matriz geradora de $C_{1}, \operatorname{com} k$ linhas e $n$ colunas, e a partir desta são geradas todas as palavrascódigo de $C_{1}$.

2. Particionamos o conjunto das $n$-uplas binárias em classes laterais do espaço vetorial $n$-dimensional em relação a $C_{1}$, i.e., construímos o arranjo padrão segundo $C_{1}$.

3. Para cada classe lateral obtemos o conjunto $S_{2} C_{1}=$ $\left.\left\{\mathbf{x}_{3} \in C_{1} ; \mathbf{x}_{3} \cdot\left(\mathbf{x}_{1} \oplus \mathbf{w}_{2}\right)=0, \mathbf{x}_{1} \in C_{1}\right\} \subseteq C_{1}\right\}$.

4. Tendo o conjunto $S_{v \oplus C_{1}}$ determinamos o conjunto $Z_{L^{\circ} \odot C_{1}}=\cup_{i=0}^{m-1} Z_{u_{2}} \subseteq 2 \oplus C_{1}$, para cada classe lateral, onde $Z_{u_{2 i}}=\left\{w_{2} x_{3} \in v \in C_{1}, \forall x_{3} \in S_{v \ominus C_{1}}, x_{3} \neq\right.$ $0\}$.

5. Determinamos o conjunto $A \cdot \odot C_{1}$, formado por todas as palavras-código pertencentes a $C_{2}$ em uma determinada classe lateral. O conjunto formado pelos $A_{\text {ed }} C_{1}$ 's de todas as classes laterais, acrescido de uma palavra-código em comum com $C_{1}$, será o próprio $C_{2}$. Denotaremos por $M$ o número de palavras-código de $C_{2}$.

Exemplo 2 Seja $C_{1}$ o código $(7,3)$ cuja matriz geradora $G$ é a seguinte

$$
G=\left[\begin{array}{lllllll}
1 & 0 & 0 & 0 & 0 & 1 & 1 \\
0 & 1 & 0 & 0 & 1 & 1 & 0 \\
0 & 0 & 1 & 1 & 0 & 0 & 0
\end{array}\right]
$$

$O$ código $C_{2}$ construído a partir do algoritmo de Cabral tem 54 palavras-código, mostradas na Figura 3. As taxas de transmisão de $R_{1}, R_{2}$ e $R$ são as seguintes.

$$
\begin{aligned}
R_{1} & =0.428 \\
R_{2} & =0,822 \\
R & =R_{1}+R_{2}=1,25 .
\end{aligned}
$$

000000000000011000010000001000000111000000 000010001000100000101010001110001101100000 000011001000000000111010000110001001100010 000100000100000001001001000110010101010010 000101000100100001011001001110010001010000 000110000101000101010011001000011010010101 010101101100111001110101011011010001110000 000111000101100101000011000000011110010111 010100101100011001100101010011010101110010

Figura 3. Palavras-código de $C_{2}$

A importância dos códigos fortemente ortogonais balanceados, advém do fato que através de busca exaustiva, para diversos valores dos parâmetros, verificamos que estes códigos possuem a mais alta taxa de transmissão atingível com a construção linear. Consideremos $n$ o comprimento das palavras-código e $k$ a dimensão do código $C_{1}$. Seja $M$ o número de palavras-código de $C_{2}, R_{1}$ a taxa de transmissão de $C_{1}, R_{2}$ a taxa de transmissão de $C_{2}$ e $R$ a taxa de transmissão conjunta no 2-BAC. Encontramos todas as matrizes geradoras possíveis para alguns valores de $n$ e $k$. Utilizamos então o algoritmo implementado acima para obtenção das palavras-código de $C_{2}$. Apresentamos os resultados nas tabelas seguintes:

1. $n=4$ e $k=2$

\begin{tabular}{|c|l|l|l|l|}
\hline$|G|$ & $M$ & $R_{2}$ & $R_{1}$ & $R$ \\
\hline 3 & 4 & 0.5 & 0,5 & 1 \\
\hline 9 & 6 & 0,646 & 0.5 & 1,146 \\
\hline 2 & 7 & 0.702 & 0,5 & 1.202 \\
\hline 2 & 9 & 0,792 & 0,5 & 1.292 \\
\hline
\end{tabular}

2. $n=6$ e $k=2$

\begin{tabular}{|c|c|l|l|c|}
\hline$|G|$ & $M$ & $R_{2}$ & $R_{1}$ & $R$ \\
\hline 5 & 16 & 0.667 & 0.333 & 1,000 \\
38 & 24 & 0.764 & 0.333 & 1.097 \\
40 & 28 & 0.801 & 0.333 & 1,134 \\
17 & 30 & 0.818 & 0.333 & 1,151 \\
2 & 31 & 0.826 & 0.333 & 1,159 \\
60 & 36 & 0,862 & 0,333 & 1,195 \\
68 & 42 & 0.899 & 0.333 & 1.232 \\
8 & 45 & 0,915 & 0,333 & 1,248 \\
12 & 46 & 0.921 & 0.333 & 1,254 \\
6 & 49 & 0.936 & 0.333 & 1,269 \\
\hline
\end{tabular}

\section{OUTRAS CONSTRUÇÕES DE CÓDIGOS PARA O 2-BAC}

Podemos citar uma série de trabalhos cuja finalidade é a construção de códigos para o 2-BAC, por exemplo [4][6][10]. Kasami e Lin [6] apresentaram um método para construção de pares de códigos $\delta$-decodificáveis. Dois códigos binários $C_{1}$ e $C_{2}$ são $\delta$-decodificáveis $(\delta>0)$ se e somente se, para quaisquer dois pares distintos de énuplas $(x, w)$ e $\left(x^{\prime}, w^{\prime}\right)$ em $C_{1} \times C_{2}, d_{L}\left(x+w, x^{\prime}+w^{\prime}\right) \geq \delta$. Um par $\left(C_{1}, C_{2}\right)$ é unicamente decodificável quando ele é 1-decodificável. Em 


\section{Maria de Lourdes M.G. Alcoforado e Valdemar Cardoso da Rocha Junior Códigos para o Canal Aditivo com Dois Usuários Binários}

publicação posterior Kasami e Lin [7] apresentaram um esquema para decodificação de códigos $\delta$-decodificáveis para o 2-BAC com ruído, levando em conta a linearidade e corrigindo no máximo $\lfloor(\delta-1) / 2\rfloor$ erros de transmissão, onde $\lfloor(\delta-1) / 2\rfloor$ denota o maior inteiro igual ou menor que $(\delta-1) / 2$. Ahswede e Balakirsky [10] apresentaram um método de construção de códigos binários unicamente decodificáveis $\left(C_{1}, C_{2}\right)$ para o 2-BAC, de comprimento $t n$, onde $t$ e $n$ são inteiros fixos, onde nem $C_{1}$ nem $C_{2}$ é linear. Rocha e Massey [8] estabeleceram uma condição de suficiência para a construção de códigos binários unicamente decodificáveis $\left(C_{1}, C_{2}\right)$ de peso constante para o 2-BAC, com ou sem ruído, particionando o dicionário de um código de peso constante. Esta construção foi aplicada [8] a várias famílias de códigos binários de peso constante como, por exemplo. códigos obtidos das matrizes de Hadamard, Steiner Systems, códigos derivados dos códigos Berlekamp-Justesen generalizados e códigos de Reed-Solomon. Massey [11] construiu então uma prova mais simples da construção de Rocha e Massey [8]. Rocha observou naquela ocasião que esta nova dedução não fazia uso da hipótese dos códigos envolvidos serem de peso constante, porém nunca publicou este resultado. O teorema a seguir, cujo enunciado difere muito pouco, porém de modo significativo. daquele enunciado em [11], fortalece o resultado obtido anteriormente por Massey.

Teorema 5 Sejam $C_{1}$ e $C_{2}$ códigos de bloco binários com comprimento de bloco n. com distâncias mininias de Hamming $d_{1}$ e $d_{2}$, respectivamente. Sejam

$$
\begin{aligned}
D_{\min } & =\min \left\{d_{H}(x, y): x \in C_{1}, y \in C_{2}\right\} \\
D_{\max } & =\max \left\{d_{H}(x, y): x \in C_{1}, y \in C_{2}\right\}
\end{aligned}
$$

então

$$
\max \left\{d_{1}, d_{2}\right\}+D_{\text {min }}>D_{\text {máx }}
$$

é uma condição suficiente para o par $\left(C_{1}, C_{2}\right)$ ser unicamente decodificável no 2-BAC.

Prova: Suponha. contrariando a hipótese. que $x+y=$ $x^{\prime}+y^{\prime}$. onde $x \in C_{1}, x^{\prime} \in C_{1}, y \in C_{2} . y^{\prime} \in C_{2}$ e $x \neq x^{\prime}$. Segue que.

$$
d_{H}\left(x^{\prime}, y^{\prime}\right)=\Pi_{H}^{*}\left(x^{\prime} \Phi y^{\prime}\right) \leq D_{\text {máx }}
$$

mas também,

$$
d_{H}\left(x \cdot x^{\prime}\right)+d_{H}\left(x \cdot y^{\prime}\right)=\amalg_{H}\left(x^{\prime} \partial y^{\prime}\right) \leq D_{\text {máx }}
$$

porque $x$ difere de $x^{\prime}$ ou de $y^{\prime}$ (mas não de ambos) apenas nos componentes onde $x^{\prime} y^{\prime}$ contém um " 1 ". Como $d_{H}\left(x, x^{\prime}\right) \geq d_{1}$ e $d_{H}\left(x, y^{\prime}\right) \geq D_{\min }$, nós temos

$$
d_{1}+D_{\min } \leq D_{\text {máx }}
$$

similarmente temos.

$$
d_{2}+D_{\min } \leq D_{\text {máx }}
$$

Portanto, máx $\left\{d_{1}, d_{2}\right\}+D_{\min }>D_{\text {máx }}$

\section{CONCLUSÕES}

O canal aditivo com dois usuários binários vem sendo investigado há quase trinta anos e algumas questões básicas ainda não foram respondidas satisfatoriamente como, por exemplo, a região de capacidade com probabilidade de erro zero ainda não é conhecida. Neste trabalho tivemos oportunidade de fazer a implementação computacional, na linguagem Vistual Basic, de um algoritmo proposto na literatura, mas até então não implementado, para obtenção de um código $C_{2}$ de máxima cardinalidade, a partir de um código linear $C_{1}$ e assim obtermos um código linear unicamente decodificável para o 2-BAC. Mostramos que a matriz geradora de um código fortemente ortogonal pode ser representada, de modo equivalente, como uma concatenação de matrizes mais simples e assim obtivemos uma expressão bastante concisa para o número de palavras-código de $C_{2}$. Introduzimos os códigos fortemente ortogonais balanceados e verificamos, através de busca exaustiva para diversos valores dos parâmetros, que estes códigos possuem a mais alta taxa de transmissão atingívei com a construção linear. Introduzimos também os códigos lineares de paridade repetida, para os quais foram deduzida: as expressões para o número de palavras-código de $C_{2}$. Vimos analiticamente que a classe de códigos lineares para o 2-BAC não atinge todos os pontos da região de capacidade para o 2-BAC.

Rocha [6] observou que os resultados de Weldon [5] para o 2-BAC são válidos também no caso em que ambos os códigos são não-lineares, sendo um deles sistemático, e conjecturou que a região de capacidade proibida para estes códigos não pode ser atingida com códigos unicamente decodificáveis. Dito de outra forma, o teorema de Weldon, sob esta nova interpretação, nos diz que restariam apenas códigos unicamente decodificáveis $\left(C_{1}, C_{2}\right)$, não-lineares e não-sistemáticos. cujas taxas ocupariam a região de capacidade proibida pelo referido teorema. Indo mais além, Rocha conjecturou que a região de capacidade do 2-BAC coberta pelas taxas $2-1 / \log _{2} 3<R_{1}+R_{2} \leq 1.5$ não é atingível com códigos unicamente decodificáveis, ou seja, com probabilidade de erro igual a zero. Esta conjectura ganhou mais força com o recente trabalho de Urbanke [14], lembrando que a região de capacidade livre de erros (error-free capacity) do 2 $\mathrm{BAC}$ ainda não é conhecida exatamente. Ahlswede e Balakirsky [10] e Khachatrian [12] propuseram recentemente classes de códig̣os não-lineares e não-sistemáticos, unicamente decodificáveis para o 2-BAC. As taxas de transmissão destes códigos. apesar de serem maiores do que as taxas alcançadas por outras construções anteriores, estão dentro da região de taxas atingíveis por códigos binários lineares.

Podemos concluir, portanto, que a construção de códigos lineares para o 2-BAC é importante na medida em que alcança probabilidade de erro zero. Para alcançar taxas de transmissão mais elevadas propomos o estudo de classes de códigos, não necessariamente unicamente decodificáveis, mas com probabilidade de erro pequena na decodificação, com taxas de transmissão o mais próximo possível da fronteira da região de capacidade para o 2-BAC. 


\section{REFERÊNCIAS}

[1] T. Kasami and Shu Lin, "Coding for a multiple-access channel", IEEE Trans. on Inform. Theorn, Vol. IT-22, Number 2. March 1976, pp. 129-137.

[2] H.A. Cabral, "Codificação para Canal de Acesso Múltiplo Síncrono". Dissertação de Mestrado, Departamento de Eletrônica e Sistemas, Universidade Federal de Pernambuco. Recife. Brasil, novembro de 1994.

[3] M.L.M.G. Alcoforado, "Implementação algorítmica de códigos lineares para o canal aditivo com dois usuários binários", Dissertação de Mestrado. Departamento de Eletrônica e Sistemas, Universidade Federal de Pernambuco. Recife, Brasil, dezembro de 1999.

[4] H.A. Cabral and V. C. da Rocha, Jr., "Linear code construction for the 2-user binary adder channel". IEEE Int. Symp. on Info. Theor, Whistler, Canada. 1995, pp. 497.

[5] E. J. Weldon, Jr., "Coding for a multiple-access channel". Information and Control, Vol. 36, 1978, pp. 256-274.

[6] V. C. da Rocha. Jr., "Codificação para o 2-BAC", Seminário de pesquisa, Departamento de Eletrônica e Sistemas, Universidade Federal de Pernambuco, Recife, Brasil, 1995.

[7] H.A. Cabral and V. C. da Rocha. Jr., "Coding for the 2-user binary adder channel", Relatório de pesquisa, Grupo de Pesquisa em Comunicações - CODEC, Universidade Federal de Pernambuco, 1995.

[8] V.C. da Rocha. Jr. and J. L. Massey. "A new approach to the design of codes for the binary adder channel", in Cryptography and Coding HI, (Ed. M.J. Ganley ), IMA Conf. Series, New Series Number 45. Oxford: Clarendon Press. 1993, pp. 179185.

[9] T. Kasami and Shu Lin, "Bounds on the achievable rates of block coding for a memoryless multiple-access channel", IEEE Trans. on Inform. Theory, Vol. IT-24. Number 2, march 1978, pp. 187-197.

[10] R. Ahlswede and V. B. Balakirsky. "Construction of uniquely decodable codes for the two-user binary adder channel". $I E$ EE Trans. Inform. Theor, Vol. 45. Number J. January 1999. pp.326-330.

1] J.L. Massey, "On codes for the two-user binary adder channel", Oberwolfach Information Theory Workshop. Germany, April 1992.

[12] G. H. Khachatrian. "A survey of coding methods for the adder channel", dedicated to Rudolph Ahlswede on the occasion of his 60th birthday. Comunicação privativa. 2000.

:3] 1.N. Herstein. Topics in Algebra. New York: Blaisdell Publishing Company, 1964.

:-1 R. Urbanke and Quinn Li. "The zero-error capacity region of the 2-user synchronous BAC is strictly smaller than its Shannon capacity". IEEE Trans. on Information Theory, aceito para publicação, 2001
Maria de Lourdes Melo Guedes Alcoforado nasceu em Recife em 27 de abril de 1973. Graduou-se (1995) e obteve o Mestrado (1999) em Engenharia Elétrica pela Universidade Federal de Pernambuco, onde atualmente é aluna de doutorado em Engenharia Elétrica, área de telecomunicaçōes. Trabalhou como engenheira do setor elétrico na empresa AmBev - Companhia de Bebidas das Américas (19972001 ). Tem interesses de ensino e de pesquisa em telecomunicações, em particular. aplicações de códigos para canais de acesso múltiplo e teoria da informação.

Valdemar Cardoso da Rocha Júnior nasceu em Jaboatão, Pernambuco. em 27 de agosto de 1947. Formou-se em Engenharia Elétrica. Modalidade Eletrônica, na Escola Politécnica da Fundação de Ensino Superior de Pernambuco (FESP) em 1970. Obteve o título de Ph.D. em Eletrônica pela University of Kent at Canterbury, Inglaterra em 1976. Desde 1977 é bolsista pesquisador do Conselho Nacional de Desenvolvimento Científico e Tecnológico (CNPq). tendo em 1993 alcançado o nível I-A. Desde 1976 trabalha na Liniversidade Federal de Pernambuco (UFPE), onde em 1977 fundou o Programa de Pós-Graduação em Engenharia Elétrica e foi seu Coordenador por duas vezes. Foi Chefe do Departamento de Eletrônica e Sistemas de 1992 a 1996 e em 1993 tornou-se Professor Titular. Tem atuado em várias instituições científicas no Brasil e no exterior. tendo sido Professor Convidado no Swiss Federal Institute of Technology - Zurich (1990-1992). Prof. Rocha é consultor técnico de diversas agências de fomento no Brasil, incluindo a CAPES e o CNPq. como Coordenador do Comitê Assessor de Engenharia Elétrica, Biomédica e Microeletrônica (1993-1995) e (19992001). Tem participado da organização de conferências no Brasil e no exterior, patrocinadas pelo IEEE e pela SBrT. É Sócio Fundador e atual Vice-Presidente (2000-2001) da Sociedade Brasileira de Telecomunicações, e é também sócio do IEEE. USA: Communications Society (1977) e lnformation Theory Society (1981): é sócio da Sociedade Brasileira de Matemática Aplicada e Computacional (1982) e é Fellow do Institute of Mathematics and its Applications (1992. Inglaterra). Sua área de interesse de pesquisa é teoria da informação digital aplicada. incluindo códigos corretores de erros e criptografia. 